\title{
BioéthiqueOnline
}

\section{Compte rendu critique du livre Voir son steak comme un animal mort, de Martin Gibert}

\section{Marie-Josée Drolet}

Volume 5, 2016

URI : https://id.erudit.org/iderudit/1044294ar

DOI : https://doi.org/10.7202/1044294ar

Aller au sommaire du numéro

Éditeur(s)

BioéthiqueOnline

ISSN

1923-2799 (numérique)

Découvrir la revue

Citer ce compte rendu

Drolet, M.-J. (2016). Compte rendu de [Compte rendu critique du livre Voir son steak comme un animal mort, de Martin Gibert]. BioéthiqueOnline, 5.

https://doi.org/10.7202/1044294ar
Résumé de l'article

Ce compte rendu critique offre, dans un premier temps, une synthèse des arguments présentés par Martin Gibert dans son livre Voir son steak comme un animal mort, et ce, en suivant pas à pas l'enchainement de ses arguments, chapitre par chapitre. Dans un deuxième temps, une perspective critique de cet ouvrage et une réflexion personnelle sont articulées. 


\title{
Compte rendu critique du livre Voir son steak comme un animal mort, de Martin Gibert
}

\author{
COMPTE RENDU / REVIEW \\ Marie-Josée Drolet $^{1}$
}

Reçu/Received: 8 Augt $2016 \quad$ Publié/Published: 18 Oct 2016
Éditrices/Editors: Lise Lévesque \& Hazar Haidar

2016 M-J Drolet, Creative Commons Attribution 4.0 International License

\section{Résumé}

Ce compte rendu critique offre, dans un premier temps, une synthèse des arguments présentés par Martin Gibert dans son livre Voir son steak comme un animal mort, et ce, en suivant pas à pas l'enchainement de ses arguments, chapitre par chapitre. Dans un deuxième temps, une perspective critique de cet ouvrage et une réflexion personnelle sont articulées.

\section{Mots clés}

véganisme, éthique animale, éthique environnementale, psychologie morale, philosophie politique, carnisme, spécisme

\section{Summary}

This critical review provides, first, a step-by-step synthesis of the arguments presented in each chapter of Martin Gibert's book Voir son steak comme un animal mort (Seeing your steak as a dead animal). Second, a critical perspective of the book and a personal reflection are presented.

\section{Keywords}

veganism, animal ethics, environmental ethics, moral psychology, political philosophy, carnism, speciesism

Affiliations des auteurs / Author Affiliations

${ }^{1}$ Département d'ergothérapie, Université du Québec à Trois-Rivières (UQTR), Trois-Rivières (Québec), Canada

\section{Correspondance / Correspondence}

Marie-Josée Drolet, marie-josee.drolet@uqtr.ca

\section{Remerciements}

Je remercie Syliane Charles, professeure au Département de philosophie de l'Université du Québec à Trois-Rivières (UQTR), de son invitation à une conférence, organisée par son département et donnée par Martin Gibert. Durant sa conférence sur l'utilité, voire la nécessité de l'imagination en éthique, Martin Gibert a fait référence à son livre Voir son steak comme un animal mort qu'il a alors présenté comme une application concrète de la thèse centrale de son ouvrage L'imagination en morale. Ayant trouvé la conférence stimulante et intéressante, j'entrepris donc de lire ses deux ouvrages. Merci à Syliane Charles pour cette émulation entre pairs ainsi qu'entre nos départements respectifs. Merci aussi à Mireille Lalancette et à Marie-Ėve Caty, toutes deux professeures à I'UQTR, pour nos échanges forts stimulants entourant les arguments développés dans son ouvrage sur le véganisme. Merci enfin aux réviseurs de la revue BioéthiqueOnline qui, par leurs judicieux commentaires et pertinentes suggestions, m'ont aidée à préciser ma pensée.

\section{Conflit d'intérêts}

Aucun déclaré

\section{Acknowledgements}

I wish to thank Syliane Charles, professor in the Department of Philosophy at the Université du Québec à Trois-Rivières (UQTR), for her invitation to a conference, organized by her department and given by Martin Gibert. During his conference on the utility, or even the necessity of imagination in ethics, Martin Gibert referred to his book Voir son steak comme un animal mort, which he then presented as a concrete application of the central thesis of his book L'imagination en morale. I found the conference to be stimulating and interesting, so I read his two books. Thanks Syliane Charles for this collegial and interdepartmental exchange. I also wish to thank Mireille Lalancette and Marie-Ëve Caty, both professors at UQTR, for stimulating discussions about the arguments developed in the book on veganism. Finally, I wish to thank the editors of BioéthiqueOnline who, through their judicious comments and relevant suggestions, helped clarify my thoughts.

\section{Conflicts of Interest}

None to declare 


\section{Introduction}

L'essai philosophique Voir son steak comme un animal mort consiste en un plaidoyer éthique et politique pour le véganisme [1]. Son auteur, Martin Gibert, est docteur en philosophie, enseigne l'éthique et la philosophie du droit, est chercheur affilié au Groupe de recherche interuniversitaire sur la normativité (GRIN) et rédacteur en chef de Versus Magazine. Son ouvrage d'éthique appliquée présente les principaux arguments l'ayant conduit à opter pour le véganisme. Dès l'introduction, Gibert y articule la thèse qu'il y défend et définit le concept central de sa réflexion, soit le véganisme. Comme il le précise, le véganisme est un mouvement social et politique contemporain qui combat toutes les formes d'oppression à l'endroit des animaux non-humains [1]. La thèse au cœur de l'ouvrage, formulée de diverses manières et à plusieurs endroits, s'articule ainsi : " s'il est possible de vivre sans infliger de souffrances non nécessaires aux animaux, alors nous devons le faire » (p.10). Pour l'auteur, « opter pour le véganisme plutôt que pour le végétarisme, c'est simplement faire le choix de l'efficacité et de la cohérence » (p.13). C'est à la démonstration de cette thèse que le lecteur est convié, et ce, dès l'introduction du livre. Quant à ce compte rendu, il vise à présenter une perspective à la fois critique et personnelle de cet ouvrage, après avoir effectué une synthèse des propos qui y sont articulés, et ce, en suivant pas à pas l'enchainement de ses arguments, chapitre par chapitre. Compte tenu que Gibert relate le cheminement de sa réflexion personnelle vis-à-vis le véganisme, je me permettrai à l'occasion de relier l'argumentaire qu'il développe à ma propre expérience de ce même questionnement.

\section{Mais qu'est-ce que le véganisme?}

Comme tout bon philosophe, Gibert distingue le véganisme des deux principaux concepts auxquels celui-ci est apparenté, soit le végétarisme et le végétalisme. Tandis que le végétarisme et le végétalisme sont des régimes alimentaires (où le premier consiste à manger des végétaux en s'abstenant de consommer de la viande animale et où le second désigne un régime alimentaire sans produits animaux),

le véganisme n'est pas un régime alimentaire. C'est un mouvement social [...] de résistance à l'oppression dont sont victimes les animaux que nous exploitons pour leur viande, leur lait ou leur fourrure. [...] Au-delà de l'assiette, le véganisme cherche à lutter contre toutes les formes d'exploitation et de souffrances animales : cuir, fourrure, tests médicaux, divertissement (corrida, zoo), abandon et maltraitance des animaux de compagnie, etc. Autrement dit, alors qu'on peut être [végétarien ou] végétalien pour des raisons de santé personnelle, on est toujours végane pour des raisons politiques et morales (p.10).

Maintenant que le concept central de l'ouvrage de Gibert est clarifié, je vais présenter une synthèse des propos qu'il y développe, avant d'articuler une perspective critique personnelle de ceux-ci.

\section{Compte rendu de l'ouvrage}

L'ouvrage de Gibert, à la jaquette originale et accrocheuse, comprend quatre chapitres. Après avoir énoncé en introduction sa thèse et circonscrit le concept de véganisme, l'auteur y décline l'argumentaire qui appuie sa thèse, suivant quatre chapitres distincts mais complémentaires. Au chapitre 1, il présente des arguments issus de l'éthique animale pour soutenir le véganisme. Au chapitre 2, il enchaine avec des arguments en provenance de l'éthique environnementale pour justifier ce choix. Au chapitre 3, il expose des arguments propres à la psychologie morale pour expliquer les résistances humaines à opter pour le véganisme. Enfin, au chapitre 4, il présente l'idéologie carniste et tente de la déconstruire, en développant des arguments philosophico-politiques pour justifier le véganisme. 


\section{Les arguments de l'éthique animale}

Au premier chapitre, l'auteur présente une synthèse du consensus actuel en éthique animale d'après lequel « il est mal d'élever et de tuer des animaux lorsque cela n'est pas nécessaire » (p.47-48). Aussi, il montre en quoi ce consensus implique le véganisme. Il débute en rappelant la vision scientifique contemporaine de l'animal, suivant laquelle il y aurait une différence de degré (pas de nature) entre l'être humain et l'animal. Les animaux sont des êtres conscients, voire sentients. "Déclarer que les animaux sont conscients, c'est reconnaître qu'ils peuvent ressentir - on dit d'ailleurs aussi qu'ils sont sentients. La conscience animale est donc la capacité à ressentir du plaisir, de la douleur et des émotions [et] d'autres types d'états mentaux » (p.21-24). II s'ensuit que "les animaux ont intérêt à ne pas souffrir " (p.25) et à persister dans l'existence, car "l'intérêt à vivre participe à la définition du bien-être » (p.41).

Gibert précise ensuite ce qu'est l'éthique animale, fait une courte histoire de cette branche de l'éthique appliquée, de La libération animale [2] de Singer à aujourd'hui et présente les postures théoriques propres aux trois principales familles de théories éthiques normatives que sont l'éthique des vertus, le déontologisme et le conséquentialisme. II rappelle que l'éthique des vertus est centrée sur l'agent moral et ses caractéristiques, que le déontologisme réfléchit en termes de devoirs de l'agent moral et droits du patient moral et, enfin, que le conséquentialisme analyse les conséquences de l'action de l'agent moral sur le bien-être des êtres sentients concernés par l'action, que ceux-ci soient humains ou non-humains. Ainsi, tandis que l'éthicien des vertus estime qu'une personne vertueuse évite d'infliger inutilement de la souffrance animale, car celle-ci a de la compassion pour tout être vivant, le déontologue non kantien estime, pour sa part, que l'être humain a des devoirs envers les animaux, car ceux-ci sont porteurs de droits éthiques, alors que le conséquentialiste propose un altruisme effectif au nom du bien-être animal. Qu'il s'agisse du "refus de la cruauté » (p.34) articulé par des éthiciens des vertus, "du respect des droits » (p.34) des animaux proposé par des abolitionnistes déontologues ou du combat " contre les souffrances [animales] inutiles " (p.34) mené par des conséquentialistes, ces trois approches en éthique animale convergent : elles revendiquent un plus grand respect et un meilleur traitement des animaux, car elles visent des rapports plus justes entre l'être humain et l'animal. Tel est le consensus en éthique animale qui soutient la thèse de l'auteur.

Gibert clôt ce premier chapitre en réfutant deux objections formulées contre ce consensus en éthique animale, soit l'objection du gastronome et celle de l'omnivore consciencieux. Contre la première, il affirme qu'elle ne tient pas la route dans le calcul de l'utilité, car le plaisir de l'être humain à manger un animal ne peut justifier ni la souffrance animale ni la mort d'animaux. Contre la seconde, il conclut que bien qu'il soit moins justifié de se préoccuper du bien-être des animaux en leur procurant des élevages moins cruels, il reste que la finalité des élevages demeure l'abattage, soit la mise à mort prématurée de l'animal sans nécessité, ce qui ne saurait être justifié.

\section{Les arguments de l'éthique environnementale}

Au second chapitre, Gibert articule des raisons issues de l'éthique environnementale pour soutenir le véganisme. Après avoir fait un état des lieux du réchauffement climatique et de la crise écologique qui secouent le monde contemporain, il énumère six raisons qui expliqueraient notre "inertie climatique » (p.74). Pour ce faire, il se tourne vers la psychologie environnementale pour comprendre ce phénomène. Gibert attribue notre passivité collective aux limites de la cognition humaine, à notre tendance à nous comparer aux autres, à l'effet de certaines idéologies sur nos attitudes et nos comportements, aux efforts que demande le changement d'habitudes de vie, à certaines émotions comme la défiance envers les autres ou le déni de la réalité ainsi qu'au fait d'opter pour des actions peu efficaces, voire inefficaces au détriment d'actions efficaces. En bref, en dépit des nombreuses preuves scientifiques des changements climatiques qui mettent en péril l'environnement et, par extension, la vie, ces raisons contribueraient à expliquer notre inertie collective. Après avoir présenté ces raisons, il explique le lien entre le réchauffement climatique et le véganisme. 
Sur ce sujet, il mentionne que l'industrie animale serait responsable de $14,5 \%$ des émissions globales de gaz à effet de serres, ce qui est supérieur aux émissions occasionnées par les transports qui seraient responsables de $11 \%$ de ces émissions. La déforestation, la fertilisation des champs, la digestion des ruminants productrice de méthane, l'entretien des exploitations, le transport des produits animaux et leur congélation consomment des énergies fossiles. C'est dire que l'empreinte écologique des êtres humains varie en fonction de leur régime alimentaire. Celle d'un carnivore serait deux à trois fois supérieure à celle d'un végane. En plus de participer au réchauffement climatique, Gibert rappelle que l'industrie animale consomme une grande quantité d'eau potable, pollue les sols, les cours d'eau et les nappes phréatiques, contribue à la réduction de la biodiversité (en soutenant les monocultures et la déforestation) et est source de gaspillage, car on peut nourrir un bien plus grand nombre d'êtres humains avec des végétaux qu'avec des animaux. "Lorsque près d'un milliard d'humains sont sous-alimentés, il y a quelque chose d'aberrant à un tel gaspillage » (p.92), soulignet-il. Gibert termine ce second chapitre ainsi : « parmi toutes les règles liées à l'alimentation, cultiver la disposition au véganisme est sans conteste celle dont on peut attendre les meilleures conséquences pour la préservation de l'environnement » (p.102).

\section{Les arguments de la psychologie morale}

Au troisième chapitre, l'auteur s'appuie sur ses connaissances en psychologie morale pour expliquer ce qu'il nomme le paradoxe de la viande qui serait lié à la banalisation de l'exploitation animale. Pour ce faire, il utilise la notion de dissonance cognitive. « En psychologie morale, la dissonance cognitive désigne l'inconfort mental qui vous saisit lorsque deux de vos croyances, ou une croyance et un comportement, sont en contradiction. Or, pour fuir cette dissonance, l'esprit humain est capable d'habiles stratégies d'évitement »(p.110). Gibert précise qu'il y aurait trois manières de réduire ou d'annuler une dissonance cognitive, soit : 1) de modifier son comportement, 2) de réviser une de ses croyances ou l'importance accordée à celle-ci ou 3) de renforcer son comportement en ajoutant de nouvelles cognitions conciliantes, c'est-à-dire des croyances qui contribuent à réduire ou annuler la dissonance cognitive. Comme il le précise, la cognition humaine aurait besoin de cohérence interne. Or, l'être humain aime en général les animaux ou souhaite la préservation de l'environnement. Pourtant, une majorité consomme des produits animaux. Tel est le paradoxe de la viande. II s'agit, estime Gibert, d'une dissonance cognitive. Tandis que les véganes ont, pour annuler celle-ci, décidé de changer leurs comportements (option 1), la majorité a développé une gamme de cognitions conciliantes pour tenter de la réduire (option 3). Tel est l'objet de ce chapitre, à savoir de nommer et d'expliquer huit cognitions conciliantes, tout en les réfutant les unes après les autres. L'auteur examine par exemple les cognitions suivantes: "Je sais que les animaux souffrent... mais j'aime mon steak » (p.115), " nous avons besoin de protéines animales » (p.124) ou encore " on pourrait avoir du lait et des œufs sans souffrance » (p.140). Ce faisant, il montre que ces stratégies visant à réduire le paradoxe de la viande reposent sur un déni des faits ou se révèlent fallacieuses, voire sophistiques.

Gibert clôt ce chapitre en discutant du carnisme qui correspond à une idéologie violente, invisible, arbitraire et imposée qui serait liée à un « système de croyances [qui] nous conditionne à trouver normal, naturel et nécessaire de manger des produits animaux » (p.150). Cette idéologie chosifie les animaux, les désindividualise et les divise en deux groupes essentiellement distincts : les animaux de compagnie et ceux d'élevage. Le carnisme serait une idéologie incarnée, en ceci qu'il correspondrait au "spécisme appliqué à nos habitudes de consommation »(p.153). Cette idéologie viserait à annuler les dissonances cognitives reliées au paradoxe de la viande. Ce faisant, elle empêche de percevoir le fait de consommer des produits animaux comme un problème éthique. Autrement dit, le carnisme "déforme notre perception morale. II est l'écran idéologique qui dissimule la réalité de l'exploitation [animale] » (p.155), la lunette qui empêche de voir son steak comme un animal mort. 


\section{Les arguments de la philosophie politique}

Au quatrième et dernier chapitre, l'auteur poursuit sa condamnation de l'idéologie carniste qui fait du spécisme une politique de la domination de l'animal par l'être humain et montre " que le véganisme n'est pas seulement un style de consommation ou un mode de vie, c'est un mouvement social, politique et moral qui s'intègre dans un refus plus vaste des oppressions »(p.158-159). Plus précisément, dans ce chapitre, Gibert s'intéresse à la dimension politique du véganisme et défend que le véganisme est un humanisme. Pour parvenir à faire cette démonstration, il s'intéresse à l'humanisme de Sartre et à celui de Descartes. Ce faisant, il montre que l'humanisme sartien et cartésien (tout comme l'humanisme chrétien) sont liés à une rupture métaphysique entre l'être humain et l'animal, selon laquelle l'être humain serait métaphysiquement distinct de l'animal et supérieur à celui-ci. Ainsi, ce type d'humanisme qui présuppose la thèse de l'exception humaine se révèle spéciste. Ce n'est donc pas en ce sens que le véganisme est un humanisme, car comme le précise Gibert, le spécisme « est la position éthique qui soutient que je ne dois de considération morale qu'aux membres de ma propre espèce » (p.168). Plus encore, "le spécisme est une forme de suprématisme humain » (p.168). Ces précisions sur l'humanisme sartrien et cartésien amènent Gibert à distinguer deux types d'humanismes, soit l'humanisme exclusif et l'humanisme inclusif. Tandis que le premier (celui de Sartre et de Descartes) exclut les animaux du cercle de la moralité, le second au contraire les inclut. C'est donc en ce second sens que le véganisme est un humanisme, car il considère, par de-delà l'appartenance à une espèce, l'intérêt de tout individu sentient à ne pas souffrir, à vivre et à ne pas être exploité.

L'humanisme "inclusif » désigne un ensemble de valeurs, de normes et de vertus morales qui sont à la base d'une extension constante du cercle de la moralité. II défend l'égalité, la liberté et la solidarité et se préoccupe des plus vulnérables. II est inclusif, car il ne restreint pas a priori le champ de l'application de ces valeurs. C'est l'humanisme de Voltaire et de Rousseau, de Jeremy Bentham et de John Stuart Mill, de Martin Luther King et de Gandhi (p.173).

Cet humanisme inclusif est celui des véganes. Ainsi, il serait faux d'affirmer que les véganes sont des misanthropes. Au contraire, être véganes, c'est élargir le cercle de la moralité à un plus grand nombre d'êtres sentients. Plus encore, ce serait manifester une plus grande empathie que l'omnivore à l'endroit des animaux et des êtres humains, car « plus les gens se soucient des animaux, plus ils se soucient des humains » (p.182).

Gibert discute ensuite de l'histoire de l'oppression des animaux en parallèle avec d'autres types d'oppressions qui marquent encore de nos jours l'humanité comme le racisme (race), le sexisme (genre), le capacitisme (handicap), l'hétérosexisme (orientation sexuelle), le classisme (classe sociale) et l'âgisme (âge). L'intersectionnalité, qui correspond à un domaine d'études en sociologie s'intéressant à "l'intersection entre différentes formes d'oppressions » (p.188), aide à comprendre les dimensions politiques de l'oppression animale, car elle permet de repérer les croissements qui se présentent entre les différents types d'oppressions. Par exemple, les femelles animales sont davantage exploitées que les mâles. Ainsi, " on ne peut comprendre adéquatement la réalité des oppressions sexiste et spéciste qu'en comprenant leur enchevêtrement » (p.191). Gibert montre aussi plusieurs parallèles pouvant être établis entre le spécisme et le racisme. " Bref, tout comme l'analogie entre espèce et sexe consolide l'oppression patriarcale, l'analogie entre espèce et race permet souvent de renforcer le racisme " (p.194). C'est pourquoi le spécisme doit se penser en intersectionnalité, estime-t-il. Car cela permet de cerner plus aisément les privilèges que procurent le système des oppressions et, éventuellement, de mieux les combattre. C'est pourquoi « le mouvement végane devrait travailler de concert avec les autres mouvements de libération » (p.202), argue-t-il, car pour déconstruire un système, il faut s'attaquer à ses articulations. En bref, " si le véganisme est un humanisme, c'est parce que les discriminations s'abreuvent aux mêmes sources »(p.214). Pour conclure son ouvrage, Gibert rappelle que pour parvenir à élargir le cercle de la moralité, encore fautil percevoir les systèmes d'oppressions. Dans cet essai philosophique, son objectif était précisément de faire voir l'oppression dont sont victimes les animaux non-humains. 


\section{Perspective critique}

\section{Ton et style}

Écrit tantôt au « je », tantôt au « il », l'ouvrage de Gibert oscille entre un ton personnel, intime et subjectif, d'une part, et un ton informationnel, factuel et objectif, d'autre part. Cette mixité d'approche dans le style de l'écriture est un choix intéressant, en ceci que le lecteur est convié, dès les premières pages, à suivre le processus cognitif et affectif qu'a vécu l'auteur lorsqu'il fut exposé aux arguments qui l'ont amené à délaisser le carnisme pour le véganisme. Ce faisant, le lecteur suit pas à pas les différentes étapes traversées par l'auteur.

Usant parfois de confidences sur sa vie personnelle et d'humour, le ton et le style d'écriture ont l'avantage de ne pas être lourds, et ce, sans négliger le contenu philosophique au fondement de la thèse qu'il défend. Ainsi, Gibert présente un ensemble de données scientifiques (en provenance de disciplines telles que la biologie animale, les sciences de l'environnement, la psychologie environnementale, la psychologie cognitive, etc.) qui appuie son propos et qui étaye sa thèse de manière somme toute convaincante. Sans assommer le lecteur avec ces données, le fait qu'il adopte par moment un ton proche de la confidence permet au lecteur (qui partage les mêmes biais cognitifs que ceux de l'auteur ou des sensibilités éthiques similaires à ce dernier) de s'identifier, dans une certaine mesure, à lui. A contrario, le lecteur qui ne partage pas les biais cognitifs ou les sensibilités éthiques de Gibert, trouvera peut-être ce ton intime et personnel inapproprié, car trop subjectif, trop anecdotique. II estimera peut-être que cette alternance entre un style personnel et un autre informationnel mine l'objectivité de l'ouvrage.

Somme toute, le fait que l'auteur parte de sa propre expérience singulière pour réfléchir comme philosophe à cette problématique éthique est à la fois habile (car on peut se reconnaître comme agent moral réfléchissant à cette problématique éthique), pédagogique (car on suit les étapes qu'un agent moral à la fois sensible et raisonnable peut être appelé à traverser) et pertinente (car on est à même de découvrir ou de redécouvrir plusieurs arguments soutenant le véganisme). De fait, ce ton intimiste crée une proximité avec le lecteur, dans la mesure où ce lecteur perçoit une parenté affective et intellectuelle avec l'auteur. Si par contre cette parenté est absente, cette stratégie argumentative peut possiblement nuire aux propos de l'auteur.

En ce qui me concerne, je n'étais pas du tout familière avec les arguments développés par Gibert dans son ouvrage. En ce sens, je peux dire que ce livre m'a réveillée de mon sommeil dogmatique largement dominé par l'idéologie carniste. Gibert m'a fait voir des réalités éthiques pertinentes qui n'étaient pas du tout saillantes pour moi auparavant. Cet ouvrage a changé ma vision du rapport de l'animal humain à l'animal non-humain que je perçois désormais comme une relation de domination et d'exploitation, ce qui n'était pas vraiment le cas auparavant. Bien que je percevais avant ma lecture certains aspects de cette relation de pouvoir, mes biais cognitifs spécistes et carnistes étaient très puissants et me maintenaient dans une grande ignorance et une inertie par rapport à ces enjeux. Sans être végane aujourd'hui (quoiqu'aspirante végane ou provégane), j'ai réduit radicalement ma consommation de viande et de produits animaux. En bref, la lecture du livre de Gibert a changé plusieurs de mes attitudes et mes comportements au quotidien.

\section{Argumentaire et structure du texte}

L'organisation faite par Gibert de ses arguments est à la fois logique et pédagogique. Comme rapporté dans le résumé présenté plus tôt, quatre chapitres distincts mais complémentaires lui permettent de présenter quatre familles d'arguments soutenant le véganisme. II est rare que des ouvrages en éthique animale ratissent si largement, c'est-à-dire développent une gamme aussi variée et complémentaire d'arguments. Telle est une autre force de l'ouvrage de Gibert.

Cela dit, tant qu'à couvrir aussi largement les arguments soutenant le véganisme, il est dommage que Gibert ait exclu les arguments nutritionnels, car ce sont généralement eux qui sont d'emblée invoqués 
lorsqu'on discute du véganisme avec un auditoire peu familier avec l'éthique animale, l'éthique environnementale, la psychologie morale ou la philosophie politique. En effet, dès l'introduction du livre, Gibert note au passage la pertinence nutritionnelle d'opter pour le véganisme, sans toutefois insister sur ce type d'arguments. Pour lui, le devoir d'être végane n'est pas lié et ne doit pas l'être au goût que l'on peut avoir ou pas pour la viande ou les produits animaux comme le fromage ou le cuir. La décision d'opter pour le véganisme doit transcender nos préférences et nos intérêts personnels d'où son choix d'écarter les arguments nutritionnels. Or ces arguments - certes reliés à nos préférences et intérêts personnels, voire à ceux de ses enfants lorsque l'on est parent - auraient été un complément nécessaire à son argumentaire. Car pour parvenir à convaincre une personne (voire une communauté) de modifier son régime alimentaire, les arguments nutritionnels ne peuvent pas être évacués. Ainsi, un chapitre sur les nombreux avantages pour la santé humaine d'opter pour le véganisme aurait bonifié son propos et contribué à déconstruire moult mythes entourant lesdits désavantages de délaisser les protéines animales.

II n'en demeure pas moins que ce livre est un incontournable. Sa pertinence repose non seulement sur la justesse de la thèse qui y est défendue, mais également sur la qualité de l'argumentation. En environ deux cent pages, Gibert réussit à synthétiser un ensemble assez considérable de connaissances de domaines aussi variés que l'éthique animale, l'éthique environnementale, la psychologie environnementale, la psychologie morale, la neuropsychologie, la philosophie politique, l'histoire et la sociologie. La grande originalité de cet ouvrage est précisément de sortir du champ usuel de la philosophie. La pluridisciplinarité de son approche enrichit son propos et lui donne des assises empiriques pertinentes. Somme toute, son ouvrage rend visible l'oppression dont sont victimes les animaux non-humains. Gibert espère ainsi réveiller de leur sommeil dogmatique maints philosophes contemporains, confie-t-il. II est vrai d'ailleurs qu'il existe un silence éthique entourant l'exploitation animale en philosophie, à tout le moins dans le monde francophone.

\section{Vocabulaire éthique}

En sortant des plates-bandes conceptuelles usuelles de la philosophie, Gibert utilise des notions en provenance de divers domaines scientifiques comme la psychologie et la sociologie qui contribuent à enrichir son propos. Par exemple, lorsqu'il traite des dissonances cognitives, des cognitions conciliantes ou de l'intersectionnalité, ces notions lui permettent de développer des arguments originaux et rarement discutés en philosophie, mais ô combien pertinents. Ces notions inscrivent son propos dans des réalités tangibles que des scientifiques de divers domaines ont documentées, ce qui apporte de la scientificité à son argumentaire. Les philosophes auraient tort de négliger ce type d'arguments.

Cela dit, Gibert aurait pu reconnaître la distinction entre l'éthique et la morale, qui sont deux disciplines semblables, mais néanmoins distinctes. Bien que souvent amalgamées par les philosophes contemporains, ces notions ont avantage à être distinguées parce qu'elles réfèrent à des réalités différentes. En effet, tandis que l'éthique est une discipline philosophique, la morale relève des us et coutumes, voire des traditions religieuses [3,4]. Ainsi, si la morale (les mœurs) actuelle avalise le spécisme et le carnisme ordinaires, l'entreprise de Gibert est éthique, car par l'entremise de son arsenal philosophique d'essence critique elle remet en question, voire condamne la morale actuelle qui légitime le suprématisme de l'animal humain sur l'animal non-humain. Somme toute, l'ouvrage de Gibert entend précisément critiquer la morale actuelle qui est intimement liée à une conception courante d'humanisme spéciste sur laquelle s'appuient la domination et l'exploitation animales. Cette morale, cet humanisme empêche une majorité d'êtres humains de percevoir ce système suprématiste.

\section{Conclusion}

En conclusion, Voir son steak comme un animal mort est un essai philosophique défendant le véganisme. Son auteur, Martin Gibert, y articule quatre types d'arguments pour étayer son propos, 
soit des arguments reliés à l'éthique animale, à l'éthique environnementale, à la psychologie morale et à la philosophie politique. Adoptant un ton très personnel et un style accessible, cet ouvrage d'éthique appliquée, qui mérite qu'on s'y attarde, part de l'expérience personnelle de l'auteur pour conduire, pas à pas, le lecteur à la découverte ou à la redécouverte d'arguments soutenant la pertinence éthique du mouvement anti-oppressionniste qu'est le véganisme. Mettant à jour le paradoxe de la viande et la banalisation de l'exploitation animale, déboulonnant l'idéologie carniste, ce livre constitue une excellente introduction au véganisme, ce mouvement éthique et politique " qui lutte pour la justice animale, sociale et environnementale " (endos du livre). Je le recommande sans hésitation, car les arguments qui y sont développés sont non seulement pertinents d'un point de vue éthique, ils sont aussi à même de transformer positivement notre rapport aux animaux non-humains.

\section{Références}

1. Gibert M. Voir son steak comme un animal mort. Véganisme et psychologie morale. Montréal. Lux Éditeur; 2015.

2. Singer P. La libération animale. Paris. Payot; 1975.

3. Legault G. Professionnalisme et délibération éthique. Québec, Presses de I'Université du Québec; 2008.

4. Després $P$. Philosopher à partir des textes : une introduction à la philosophie et à la rationalité. Québec, Beauchemin; 2004

\section{Lectures recommandées}

Martin Gibert puise à plusieurs publications phares de l'éthique animale, l'éthique environnementale, la psychologie morale et la philosophie politique. Les suggestions de lecture qui suivent ne sont que quelques-unes parmi l'ensemble des nombreuses références que Gibert convoque pour étayer ses arguments.

\section{Éthique animale}

- $\quad$ Singer P. La libération animale. Paris. Payot; 1975.

- Jeangène Vilmer JB. L'éthique animale. Paris, Presses Universitaires de France; 2011.

- Low P. et al. Déclaration de Cambridge sur la conscience. Cahiers antispécistes. 2012;35.

- Francione G. Eat like you care: an examination of the morality of eating animals. Logan, Exempla Press; 2013.

\section{Éthique environnementale}

- Gifford R. The dragons of inaction: psychological barriers that limit climate change mitigation and adaptation. American Psychologist. 2011;66(4):290-302.

- Scarborough P et al. Dietary greenhouse gas emissions of meat-eaters, fish-eaters, vegetarians and vegans in the UK. Climate change. 2014;125(2):179-192.

- Nicolaas G. Pierson Foundation, Meat the truth. Productions Alalena; 2007.

- A.U.M. Films. Cowspiracy. Kip Andersen \& Keegan Kuhn, producers; 2014.

\section{Psychologie morale}

- $\quad$ Gibert M. L'imagination en morale. Paris. Herman, L'avocat du diable; 2014.

- $\quad$ Gibert M. Imagination et perception morale. Montréal, Université de Montréal; 2012.

- Festiger L, Riecken HW, Schachter S. When prophecy fails: A social and psychological study of a modern group that predicted the destruction of the world. Minnesota, University of Minnesota Press; 1956.

- Croyle R, Cooper J. Dissonance arousal: physiological evidence. Journal of Personality and Social Psychology. 1983;45(4):782-791. 


\section{Philosophie politique}

- Harari YN. Sapiens: A brief history of humankind. Penguin Random House; 2014.

- Donaldson S, Kymlicka W. Zoopolis : Une théorie politique des droits des animaux. Paris, Alma Éditeur; 2016.

- Adams CJ. The sexual politics of meat. Bloomsbury Academic; 2015.

- Giroux V. Du racisme au spécisme: l'esclavagisme est-il moralement justifiable? Ithaque. 2007;2(1):79-107. 